\title{
Knowledge of adult patients with cystic fibrosis about their illness
}

\author{
S P Conway, M N Pond, A Watson, T Hamnett
}

\begin{abstract}
Background - Adult patients need to understand their illness if the locus of control is to move from doctor to patient. Previous studies have shown important misconceptions and gaps in patients' knowledge about cystic fibrosis.

Methods - Patients were invited to complete a multiple choice questionnaire covering all major aspects of cystic fibrosis. The questionnaire score was compared with a predicted score derived from the consultant, cystic fibrosis fellow, nurse, and physiotherapist ratings of patient knowledge. Data were obtained to provide a comprehensive patient profile and disease severity score. Both scores were tested for any associations with patient characteristics.
\end{abstract}

Results - Although patients had good general knowledge about the aspects of cystic fibrosis that impacted most on their daily lives - that is, respiratory and gastrointestinal problems - important gaps and misconceptions in these areas were still present. Knowledge and understanding of genetic and reproductive issues and the less common complications of cystic fibrosis were only moderate. Older more severely affected patients, and those who had more contact with the hospital caring team, had better multiple choice questionnaire knowledge scores. Professional carers were poor judges of the knowledge of individual patients.

Conclusions - Important gaps persist into adult life in the knowledge patients with cystic fibrosis have about their illness. $\mathrm{Ob}-$ jective assessment of these deficits is required so that each patient can be counselled according to his or her needs. (Thorax 1996;51:34-38)

Keywords: cystic fibrosis, adults, patient knowledge.

Regional Adult Cystic Fibrosis Unit, Seacroft Hospital, York Road, Leeds LS14 6UH, UK

S P Conway

$M$ N Pond

A Watson

T Hamnett

Correspondence to:

Dr S P Conway.

Received 3 May 1995 Returned to authors 11 July 1995

Revised version received

8 September 1995

Accepted for publication

12 September 1995 haphazard patient education. ${ }^{3}$ As the children grow and their understanding increases, parents and professional carers may neglect to upgrade appropriately information given to the younger patient. ${ }^{4}$ In addition, patients themselves may not press for information ${ }^{56}$ which might shatter their edifice of denial, and hence their ability to cope with an eventually fatal disease. Consequently, adult patients may still have significant gaps in their knowledge about cystic fibrosis. Greater knowledge about their illness is essential for the greater sense of control and independence that must accompany the patients' graduation to adult life. We need to appraise their state of knowledge so that we can tell them what they need to know and correct any misconceptions. ${ }^{6}$ The aim of the study was threefold: (1) to determine the level of knowledge about cystic fibrosis of a large group of adult patients and the ability of doctor, nurse, and physiotherapist to predict each patient's knowledge; (2) to test for any relationship between sociodemographic factors and patient knowledge; and (3) to determine the main sources of patients' knowledge.

\section{Methods}

Patients were asked to complete a 25-stem (120 part) multiple choice questionnaire covering the following areas of cystic fibrosis: lung infection (nine questions), gastrointestinal problems (five questions), reproduction and sexuality, lung complications, general and miscellaneous (two questions each), diabetes mellitus, liver problems, nasal polyps, lung transplant, genetics (one question each). (The full questionnaire is available on request.)

The consultant or registrar gave the questionnaire to patients when they were in a stable clinical condition at outpatients or at the end of a course of intravenous antibiotic therapy. Questions were answered as "true", "false", or "don't know", scoring " +1 " for a correct answer, " -1 " for an incorrect answer, or " 0 " for "don't know". The latter response was included to reduce guessing. Each of the questions consisted of four or five related parts so that patients scored between \pm 4 or 5 on each question. The questionnaire was independently approved by a consultant specialist in cystic fibrosis, a professor of medicine, a cystic fibrosis research fellow, and a social worker as being representative of what adults with cystic fibrosis might be reasonably expected to know, and was written in an easily understood and unambiguous style.

Data were obtained on each patient to provide a patient profile: 
(1) age;

(2) sex;

(3) social class;

(4) employment status;

(5) number of outpatient visits in preceding year;

(6) time as an inpatient in preceding year;

(7) number of inpatient treatments in preceding year;

(8) $\%$ weight/height and $\%$ predicted $\mathrm{FEV}_{1}$ (averaged over outpatient visits over the preceding year);

(9) Chrispin-Norman ${ }^{7}$ and ShwachmanKulczycki ${ }^{8}$ scores;

(10) patient and doctor (SPC) ratings of disease severity on a visual analogue scale.

Data from (7) to (10) were used to calculate an overall disease severity score.

The consultant, cystic fibrosis fellow, specialist nurse, and physiotherapist predicted the patients' knowledge scores and rated them in quartiles. Correlations between these predicted quartiles and the quartiles generated by the questionnaire were measured by Goodman and Kruskal's gamma." An overall "predicted score" was derived from the average of the four

Table 1 Ranking of median scores for individual questions*

\begin{tabular}{lll}
\hline Question content & Median score & Possible score \\
\hline Predisposing factors for respiratory infections & 5 & 5 \\
Symptoms for respiratory exacerbations & 5 & 5 \\
Male sexual status & 4 & 4 \\
Dietary requirements & 5 & 5 \\
Use of bronchodilators & 5 & 5 \\
Aspects of physiotherapy & 5 & 5 \\
Infective agents and oral antibiotic therapy & $4 \cdot 5$ & 5 \\
Abdominal symptons & 4 & 5 \\
Treatment for malabsorption & 3 & 4 \\
Intravenous antibiotic use & $3 \cdot 5$ & 5 \\
CF gene, inheritance issues and gene therapy & 3 & 5 \\
Haemoptysis & 3 & 5 \\
Reasons for malabsorption & 3 & 5 \\
Lung transplantation & 3 & 5 \\
Diabetes mellitus & 2 & 4 \\
Corticosteroid for relief of respiratory symptoms & 2 & 4 \\
Nebulised antibiotic therapy & $2 \cdot 5$ & 5 \\
Female sexuality and reproduction & 2 & 5 \\
Antibiotic use (general) & 2 & 5 \\
Nasal polyps & 1 & 4 \\
Pneumothorax & 1 & 5 \\
Distal intestinal obstruction syndrome & 1 & 5 \\
Liver disease & 1 & 5 \\
\hline
\end{tabular}

*Two miscellaneous questions not included in the ranking.

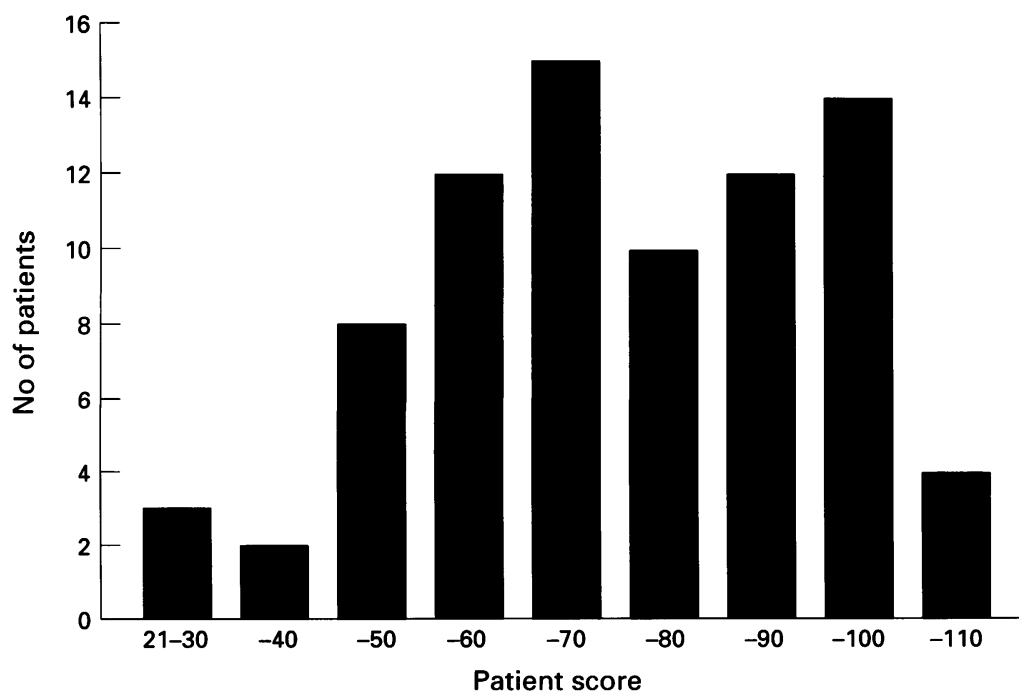

Distribution of patient scores on the multiple choice questionnaire.
Table 2 Median scores for groups of related questions

\begin{tabular}{ll}
\hline $\begin{array}{l}\text { Question content } \\
\text { (no. of related questions) }\end{array}$ & $\begin{array}{l}\text { Median/maximum } \\
\text { score (\%) }\end{array}$ \\
\hline Lung disease and infection (9) & $30 / 44(68)$ \\
Gut related problems (5) & $16 / 24(67)$ \\
Reproduction/sexuality (2) & $5 / 9(56)$ \\
Respiratory complications (2) & $4 / 9(44)$ \\
\hline
\end{tabular}

professional carers' quartile ratings. Associations between either the predicted score or the questionnaire score and patient characteristics were assessed using the WilcoxonMann-Whitney U test, the Kruskal-Wallis one way analysis of variance, or by testing the significance of the Spearman rank correlation coefficient, as appropriate.

As an addendum to the main body of the questionnaire, patients were asked to comment in their own words what the main sources of their information about cystic fibrosis had been, and whether they read the patient-directed literature on cystic fibrosis.

\section{Results}

Eighty (88\%) of 91 patients responded to the questionnaire. The median patient age was 22 years (range 15-40). There were 37 men and 43 women; $36(45 \%)$ were in full or part-time employment, $22(2 \cdot 75 \%)$ were unemployed, and $22(27 \cdot 5 \%)$ were in further education; eight $(10 \%)$ were in social class $1,17(21 \cdot 2 \%)$ were in social class $2,38(47.5 \%)$ in social class 3 , nine $(11 \cdot 2 \%)$ in social class 4 , and eight $(10 \%)$ in social class 5 .

The distribution of total scores (maximum possible 120) is shown in the figure. The minimum, median, and maximum scores were 23 , 71 , and 110 respectively. The median scores for each question are given in table 1 , and median scores for related groups of questions in table 2.

Patient knowledge about the effect of cystic fibrosis on the lungs and the gastrointestinal tract were good but nonetheless showed some important gaps in understanding - for example, all patients recognised increased sputum volume and more green sputum as indicating increased infection. Seventy seven $(96 \%)$ knew that bronchodilators "open up the airways", $74(92.5 \%)$ that a respiratory viral infection might exacerbate the condition of their chest, and that they should seek medical advice whenever suffering "common cold" symptoms. Seventy three $(91 \%)$ knew that physiotherapy needs to be done even when feeling well, 71 $(89 \%)$ that flucloxacillin is a long term antistaphylococcal agent, $69(86 \%)$ that ciprofloxacin is an effective oral anti-pseudomonal antibiotic, and $62(77.5 \%)$ that Pseudomonas aeruginosa is the most common bacterial respiratory pathogen. Seventy eight $(97 \cdot 5 \%)$ knew that they should eat the normal family meal, $75(94 \%)$ that stools are greasy because of fat malabsorption, and $72(90 \%)$ that pancreatic enzyme supplements are necessary with snacks. However, $30(37.5 \%)$ believed that nebulised antibiotics were a treatment for wheezing, $21(26 \%)$ that they should be taken 
Table 3 Correlations between cystic fibrosis carers' predicted scores with each other and with quartiles for the questionnaire score

\begin{tabular}{lllll}
\hline & Research fellow & Physiotherapist & Specialist nurse & Score \\
\hline Consultant & 0.69 & 0.68 & 0.59 & 0.33 \\
Fellow & & 0.53 & 0.62 & 0.43 \\
Physiotherapist & & & 0.50 & 0.34 \\
Specialist nurse & & & 0.60 \\
\hline
\end{tabular}

before physiotherapy, and $13(16 \%)$ that they were as effective as intravenous antibiotics in the treatment of acute exacerbations. Fifteen $(19 \%)$ were unsure whether or not a deficiency of pancreatic enzyme resulted in respiratory infection. Forty one (51\%) answered incorrectly when asked whether symptoms of severe constipation can result from too few enzymes, and $15(19 \%)$ did not know that abdominal pain and swelling are associated with taking insufficient pancreatic enzyme supplements.

Gaps were found in knowledge about reproduction and genetics; five (three men, total $6 \%$ ) did not know whether men with cystic fibrosis were sterile or not, 12 (seven men, total $15 \%)$ believed that they were not; $69(86 \%)$ knew that both parents of a child with cystic fibrosis must carry the genetic mutation and $66(82.5 \%)$ that a high risk couple have a one in four risk in each pregnancy, but $21(26 \%)$ answered incorrectly when asked whether a woman with cystic fibrosis would pass a cystic fibrosis gene to all her offspring; a further 17 (21\%) did not know.

Only $40(50 \%)$ knew that lung transplantation was followed by lifelong associated treatment and knowledge was poor about complications of cystic fibrosis. Only 49 (61\%), 43 (54\%), and $14(17 \cdot 5 \%)$ knew that the risk of haemoptysis, diabetes mellitus, and pneumothorax, respectively, increased with age. Twenty four (30\%) did not know that liver disease can occur in cystic fibrosis, $37(46 \%)$ that it can present as an enlarged liver, and only $14(17 \cdot 5 \%)$ knew that it can be associated with vomiting blood.

Correlations between the cystic fibrosis carers' predicted quartiles and the quartiles derived from the questionnaire are shown in table 3. The correlation between the predicted and questionnaire derived quartiles was $0 \cdot 48$. The physiotherapists' predictions showed a non-significant positive correlation with patient knowledge of physiotherapy, but a slight negative relationship with patient knowledge of lung disease $(p<0.05)$

Table 4 Significance of association between knowledge (questionnaire score and predicted score) and patient characteristics

\begin{tabular}{lll}
\hline & $\begin{array}{l}\text { Questionnaire score } \\
p \text { value* }^{*}\end{array}$ & $\begin{array}{l}\text { Predicted score } \\
p \text { value* }\end{array}$ \\
\hline Age & $<0.001$ (older) & $<0.001$ (older) \\
Sex & NS & NS \\
Employment status & NS & $<0.001 \dagger$ \\
Social class & 0.02 (higher) & $<0.001$ (higher) \\
Number of inpatient episodes in last year & $<0.01$ (more) & NS \\
Time as inpatient in last year & $<0.001$ (longer) & NS \\
Number of outpatient visits & NS & NS \\
Severity score & $<0.001$ (higher) & NS \\
\hline
\end{tabular}

* Where significant, knowledge was greater for stated category.

t The employment categories in descending order of median knowledge score were: student employed, unemployed.
There was a significant correlation between the severity of patients' respiratory disease as judged by higher Chrispin-Norman chest radiograph scores, and correct answers to questions both on lung disease and infection, and on physiotherapy (Spearman rank correlation coefficients $0.36(\mathrm{p}<0.001)$ and $0.27(\mathrm{p}=$ $0 \cdot 02$ ), respectively). For patients' assessment of their own disease severity and knowledge score, the Spearman rank correlation coefficient was $0.36(p<0.001)$. Correlation between the difference in the doctors' and patients' assessments of disease severity and the knowledge score was $-0 \cdot 1$ (not significant).

The results of testing for any associations between patient characteristics and either of the two knowledge scores are shown in table 4. The predictive effect of each variable remains consistent whether or not the other variables are included in a regression model, suggesting that there is no confounding effect amongst the variables measured.

Sixty one $(76 \%)$ answered the additional questions concerning the sources of their knowledge about cystic fibrosis. The majority, 41 $(67 \%)$ had learnt most from the cystic fibrosis team of specialist doctors, physiotherapists, nurses, and social workers, but $15(25 \%)$ cited other ward medical and nursing staff, $13(21 \%)$ parents, and $11(18 \%)$ other patients as their main source of information. The United Kingdom and International Adult Cystic Fibrosis Association newsletters or the Cystic Fibrosis Trust "CF News" were read by 27 (44\%).

\section{Discussion}

Overall knowledge of cystic fibrosis was good with a median questionnaire score of 71 (59\%). The mean score in the study by Nolan et al of patients $>12$ years of age was $68 \%,{ }^{3}$ and the results of Hames et $a l^{2}$ and Henley and Hill ${ }^{10}$ were similar. There was, however, a disparity in scores relating to different cystic fibrosis issues; lung disease and infection and common gastrointestinal problems scored high, reproductive issues and rarer complications scored lower - for example, median/maximum scores for questions on haemoptysis, pneumothorax, nasal polyps, distal intestinal obstruction syndrome, hepatic disease, and reproductive issues were, respectively, $3 / 5,1 / 5$, $1 / 4,1 / 5,1 / 5$, and $2 / 5$. Nolan similarly found $75 \%$ of his patients ignorant of liver and testicular problems, $57 \%$ unaware of the cause of severe constipation, and $39 \%$ not knowing the risks to respiratory health of pregnancy. ${ }^{3}$ Meghdadpour and Steele found that $30 \%$ were confused about male and/or female reproductive capacity, ${ }^{11}$ and Hames et al that only $27 \%$ were aware of the possibility of male sterility. ${ }^{2}$

We found a better understanding of genetic issues than previous studies which have reported ignorance on the probability of being affected. $^{21011}$ The facts that both parents of a child with cystic fibrosis must carry the cystic fibrosis gene and that the risk of a future affected child is one in four in each subsequent pregnancy were understood by $86 \%$ and $83 \%$ 
respectively, but only $52 \%$ realised that a mother with cystic fibrosis will pass a cystic fibrosis gene to all her children. Because some risks are well known does not necessarily mean that the basis of recessive inheritance is properly understood.

Poor knowledge about treatment may result in unintentional poor compliance with treatment regimens. Henley and Hill reported $72 \%$ patients inhaling nebulised antibiotics before physiotherapy and $17 \%$ failing to link the amount of fat in the stool with pancreatic enzyme dosage. ${ }^{10}$ We found $19 \%$ who were unaware that abdominal distension and pain are associated with inadequate pancreatic enzyme replacement, $26 \%$ who believed that nebulised antibiotics should be taken before physiotherapy, thus lessening their efficacy, and $16 \%$ who believed the latter to be as effective as intravenous antibiotics in the treatment of acute exacerbations. This misconception may delay appropriate self-referral.

There was only a weak correlation between the cystic fibrosis carers' predicted score and the questionnaire score $(r=0 \cdot 48)$, showing that the core members of the cystic fibrosis team were poor judges of individual patient's overall knowledge. The specialist nurses' greater predictive accuracy undoubtedly reflects the time they spend in direct contact with the patients, and the insufficient informal contact between doctor and patient. This is a reminder to optimise available resources. Our nurses are likely to know patients better than anyone else.

Carers may more accurately predict patient knowledge about the areas of cystic fibrosis on which they specifically advise. The physiotherapist's prediction of patient knowledge showed a non-significant positive correlation with patient knowledge about physiotherapy, though a negative correlation with patient knowledge about more general aspects of lung disease and infection. This may have important implications for intervention - that is, specialists within the team may be able to identify those patients needing more information about their own area of expertise in cystic fibrosis. We are testing this hypothesis with a pilot questionnaire designed to correlate physiotherapist, dietician, and cystic fibrosis specialist nurse predictions of patient knowledge in their own areas of treatment with patient knowledge scores on questions specific to those areas. Feedback from this study should improve carers' lack of insight into the patients' knowledge base. The latter should be regularly assessed by objective methods. Ideally any deficiencies identified should be corrected on a one to one basis. This counsel of perfection may not be achievable with existing staffing levels. We are therefore holding a series of evening interactive sessions in which specific areas of cystic fibrosis (selected after consultation with patients) are discussed, the format being a short lecture by a member of the cystic fibrosis team followed by an informal, open ended discussion. We hope that these sessions will increase patients' knowledge about their disease and highlight aspects of cystic fibrosis that are ill understood and mis- understood. Patients who choose not to attend these sessions may be expressing a wish for no further knowledge about cystic fibrosis. This may be an important part of a denial-coping mechanism and should be respected.

The adult cystic fibrosis team has no way of knowing how much patients know about their disease at the time of referral. We are therefore now asking all new patients to complete the knowledge questionnaire, the analysis of which will allow early and appropriate intervention.

Henley and Hill found no sociodemographic variable associated with patient knowledge about cystic fibrosis. ${ }^{10}$ Nolan et al reported better questionnaire performance with increasing age, higher educational level, and female sex, but found disease severity to have no predictive value. ${ }^{3}$ Both questionnaire score and predicted score in our study correlated significantly with increasing patient age and also with higher social class, perhaps reflecting the effects of a higher educational level, but we found no relationship with male or female sex. The significant association of the predicted score with employment status (students scoring highest) may indicate assessor bias. The questionnaire score, but not the predicted score, showed significant correlation with the number of inpatient episodes, time spent as an inpatient, and the severity score. The degree of respiratory disease, as defined by higher Chrispin-Norman chest radiograph scores, also showed a significant correlation with patient knowledge about lung disease and physiotherapy. This suggests that patients benefit from increased exposure to the multidisciplinary cystic fibrosis team and/or that those most severely affected actively seek more information.

There was no evidence of an association between patients' underestimation of their disease severity - that is, denial - and a poor knowledge score - that is, patients using denial as a coping mechanism not wanting to learn about their disease. Not only was there a significant correlation between patient assessment of disease severity and knowledge score, but the difference between the patient's and the doctor's assessment (negative scores corresponding to underestimation) showed a nonsignificant correlation with the knowledge score.

In conclusion, adult patients with cystic fibrosis showed good general knowledge about their illness but poor appreciation of its less common complications, poor assimilation of the rationale, if not the facts, underlying inheritance issues, and some important misconceptions about common treatments. The latter might inadvertently affect their compliance with, and the efficacy of, that therapy. Younger patients rely heavily on parents for information about cystic fibrosis. ${ }^{3}$ Our patients relied most on the cystic fibrosis specialist staff but other significant sources were ward staff, parents, and cystic fibrosis literature. Parents may be given little further information after initial consultation at the time of diagnosis ${ }^{1611}$ and wrong information may be disseminated by non-specialist sources. ${ }^{210}$ Those responsible 
for patients with cystic fibrosis should regard education of all staff and the patients' families as a high priority.

1 Canam C. Talking about cystic fibrosis within the family what parents need to know. Issues in Comprehensive Pediatric what parents need to know $1986 ; 9: 167-79$.

2 Hames A, Beesley J, Nelson R. Cystic fibrosis: What do patients know, and what else would they like to know?

3 Nolan T, Desmond K, Herlich R, Hardy S. Knowledge of cystic fibrosis in patients and their parents. Pediatrics 1986 77:229-35.

4 Glendon M. Sometimes patient teaching is as simple as child's play. Nursing 1985;July:44-6.
5 Bywater EM. Adolescents with cystic fibrosis: psychosocial adjustment. Arch Dis Child 1981;56:538-43.

6 Henley LD, Hill ID. Global and specific disease-related information needs of cystic fibrosis patients and their families. Pediatrics 1990;85:1015-21.

7 Chrispin AR, Norman AP. The systematic evaluation of the chest radiograph in cystic fibrosis. Pediatr Radiol 1974;2: 101-5.

8 Shwachman H, Kulczycki LL. Long-term study of one hundred and seventy five patients with cystic fibrosis. $\mathrm{Am}$ fDis Child 1958;96:6-15.

9 Goodman LA, Kruskal WH. Measures of association for cross-classifications, part 1. $\mathcal{F}$ Am Statist Assoc 1954;49: 732-64.

10 Henley LD, Hill ID. Errors, gaps, and misconceptions in the disease-related knowledge of cystic fibrosis patients and their families. Pediatrics 1990;85:1008-14.

11 Meghdadpour S, Steele P. Individual assessment of CF knowledge. Pediatr Pulmonol 1992;8(Suppl):A324. 